\title{
Force Pattern and Acceleration Waveform Repeatability of Amateur Runners +
}

\author{
Ophelie Lariviere ${ }^{1,2}$, Thomas Provot 1,3,*, Laura Valdes-Tamayo ${ }^{3}$, Maxime Bourgain 1,3 and \\ Delphine Chadefaux ${ }^{2,4}$ \\ 1 EPF-Graduate School of Engineering, 3 bis rue Lakanal, 92330 Sceaux, France; \\ ophelie.lariviere@edu.univ-paris13.fr (O.L.); maxime.bourgain@epf.fr (M.B.) \\ 2 Institut de Biomécanique Humaine Georges Charpak, Université Sorbonne Paris Nord, IBHGC, UR 4494, \\ F-93000 Bobigny, France; delphine.chadefaux@univ-paris13.fr \\ 3 Arts et Métiers Institute of Technology, Institut de Biomécanique Humaine Georges Charpak, IBHGC, \\ UR 4494, F-75013, Paris, France; laura.valdes@ensam.eu \\ 4 Département STAPS, Université Sorbonne Paris Nord, F-93000 Bobigny, France \\ * Correspondence: thomas.provot@epf.fr; Tel.: +33-141-134-909 \\ + Presented at the 13th conference of the International Sports Engineering Association, Online, \\ 22-26 June 2020.
}

Published: 15 June 2020

\begin{abstract}
Although accelerometers' responses during running are not perfectly understood, they are widely used to study performance and the risk of injury. To outline the typical tibial acceleration pattern during running, this study aims to investigate the repeatability of acceleration signals with respect to the ground reaction force waveforms. Ten amateur runners were asked to perform ten trials along a straight line. One participant was asked to perform this protocol over ten sessions. Tibial accelerations and ground reaction forces were measured during the stance phase. The coefficient of multiple correlation $R$ was computed to study the intra- and inter-test and subject repeatability of accelerometric and force waveforms. A good $(R>0.8)$ intra- and inter-test repeatability was observed for all measured signals. Similar results were observed for intra-subject repeatability. A good intersubject repeatability was observed only for the longitudinal acceleration and vertical and anteroposterior forces. Typical accelerometric signatures were outlined for each case studied.
\end{abstract}

Keywords: running; stance phase; accelerometer; ground reaction force; repeatability

\section{Introduction}

Running has been a growing activity for the last decade, and nowadays it accumulates more and more interest. Consequently, numerous studies have focused on a detailed description of the running mechanism in order to understand performance [1], or the appearance of injuries [2].

The biomechanical description of running has been mainly performed with experimental tools such as opto-electronic cameras or force platforms to capture kinematic and dynamic information [3]. Specifically, the vertical and anteroposterior components of the ground reaction forces (GRFs) have been used to understand the damping and propulsion mechanisms in running through the stance phase [4]. GRF signals have shown consistent waveforms and are commonly used to describe running [5]. However, although these systems are highly valuable to precisely capture running in a laboratory, they are highly restricted to investigate successive running cycles in a running field.

Today, the evolution of technology has made it possible to study running in realistic conditions, based on wearable embedded sensors [6]. These sensors have been used and evaluated many times for sports studies [7]. Specifically, accelerometers have been used to investigate the risk of injuries induced by the shocks and vibrations related to the foot/ground impacts [8]. Additionally, accelerometers 
have also been used to get insight into running kinematics [9]. However, if the link between force and acceleration signals seems possible [10], additional studies are still needed to quantify the consistence and the variability of these measures during running.

The aim of this study is to investigate and quantify the repeatability of accelerometric waveform during running. This study focused on a comparison between accelerations and GRF for an amateur runner population. It is hypothesized that (1) it is possible to extract a typical acceleration waveform during running on the basis of repeatable and reproducible protocol and that (2) it is possible to extract a typical acceleration waveform for an amateur runner population.

\section{Materials and Methods}

\subsection{Participants}

Ten recreational runners participated in this study ( 5 females and 5 males, age: $23.4+/-2.5$ years old, height: $175+/-8 \mathrm{~cm}$; mass: $68+/-8 \mathrm{~kg}$ ). Participants were rear-foot strikers and were asked to wear their own running shoes for the experiment.

\subsection{Materials}

Accelerations' signals were collected using a tri-axial accelerometer (full scale: $+/-1000 \mathrm{~g}$ ) with an embedded datalogger (S3-1000G-HA \& MWX8, Biometrics Ltd., Ynysddu, UK) at a sampling frequency of $20 \mathrm{kHz}$. The accelerometer was mounted on the distal extremity of the medial surface of the right tibia. One axis of the accelerometer was aligned with the longitudinal axis of the segment and directed upward. The accelerometer was fixed to the skin using an adhesive tape and secured using an elastic band tightened to the limit of comfort. GRF were recorded through a tri-axial force platform (OR6, AMTI, Watertown, NY, USA) at a sampling frequency of $1 \mathrm{kHz}$. The force platform was fixed at the middle of a ten meter straight line in the laboratory. Both systems were synchronized in order to record all data simultaneously.

\subsection{Protocol}

Participants were asked to run at a constant and comfortable speed $(10.9+/-1.1 \mathrm{~km} / \mathrm{h})$ along a ten meter straight line, and to run over the force platform with their right foot. Before the measurement session, an unlimited warm-up period was provided in order to familiarize the participants with the experimental context. Participants conducted as many trials as required in order to collect ten successful trials during a unique session, according to the task realization criterion (visually undisturbed running pattern and successful measurement of the force).

One of the participants was asked to perform ten sessions over ten consecutive weeks in order to study the intra- and inter-test repeatability (Study TEST). The other participant were recruited for one session in order to study the intra- and inter-subject repeatability (Study SUBJECT).

\subsection{Data Processing}

The stance phase during running is defined as the period between foot-strike and foot-off. These events are commonly defined where the vertical component of the GRF is superior to $10 \mathrm{~N}$ on rising for foot-strike and inferior to $25 \mathrm{~N}$ on descending for foot-off [11]. Consequently, the stance phase events were first extracted from the force platform signals. Then, the GRF and the tibial acceleration were segmented according to these events. Finally, the acceleration norm was computed for the ten investigated stance phases. As the stance duration was different for each participant, signals were standardized to a uniform length of 5000 sample points (representing 100\% of the stance). In order to limit the effect of the running speed on the waveform similarities, acceleration signals were normalized by subtracting the mean signal and dividing by the standard deviation (for each axis respectively) and force signals were normalized according to the weight of the participant.

The waveform similarity of each different signal was studied with a coefficient of multiple determination adapted from the methodology described by Kadaba et al. [12] to evaluate the repeatability 
of waveforms. For the study TEST, the repeatability (intra-test) and reproducibility (inter-test) of the protocol were computed with the data of the ten sessions for one participant. The intra-test repeatability coefficient $\left(R_{\text {intra-test }}^{2}\right)$ was studied comparing each trial of one session to the mean trial of this session according to the Equation (1):

$$
R_{\text {intra-test }}^{2}=1-\frac{\sum_{i=1}^{M} \sum_{j=1}^{N} \sum_{t=1}^{T}\left(Y_{i j t}-\overline{Y_{l t}}\right)^{2} / M T(N-1)}{\sum_{i=1}^{M} \sum_{j=1}^{N} \sum_{t=1}^{T}\left(Y_{i j t}-\overline{Y_{l}}\right)^{2} / M(N T-1)},
$$

where $Y_{i j t}$ is the $t$ th sample of the $j$ th trial of the $i$ th session, $T$ the number of samples per signal (5000), $N$ the number of trials per session (10) and $M$ the number of sessions (10). The inter-subject repeatability coefficient $\left(R_{\text {inter-test }}^{2}\right)$ was studied comparing all the trials of the ten sessions to the mean of all trials of all the sessions following the Equation (2):

$$
R_{\text {inter-test }}^{2}=1-\frac{\sum_{i=1}^{M} \sum_{j=1}^{N} \sum_{t=1}^{T}\left(Y_{i j t}-\bar{Y}_{t}\right)^{2} / T(M N-1)}{\sum_{i=1}^{M} \sum_{j=1}^{N} \sum_{t=1}^{T}\left(Y_{i j t}-\bar{Y}\right)^{2} /(M N T-1)} .
$$

The positive square root of the coefficient of multiple determination, called the coefficient of multiple correlation (respectively $R_{\text {intra-test }}$ and $R_{\text {inter-test }}$ for the intra- and inter-test repeatability) was used to describe the repeatability and reproducibility of accelerometric and force signals.

A similar methodology was used for the study SUBJECT for intra- and inter-subject repeatability using the data of one session for ten participants. Intra-subject repeatability coefficient $\left(R_{i n t r a-s u b j}\right)$ was studied by comparing each trial of the participant to his own mean trial (where $Y_{i j t}$ is the $t^{\text {th }}$ sample of the $j$ th trial of the $i$ th participant, $T$ is the number of samples per signal, $N$ is the number of trials per participant and $M$ is the number of participants). The inter-subject repeatability coefficient $\left(R_{\text {inter-subj }}\right)$ was studied by comparing all the trials of all the participants to the mean of all the trials of all the participant. In both studies (TEST and SUBJECT), an $R$ value greater than 0.8 was considered as good repeatability [13]. The waveform repeatability study is summarized in Figure 1.

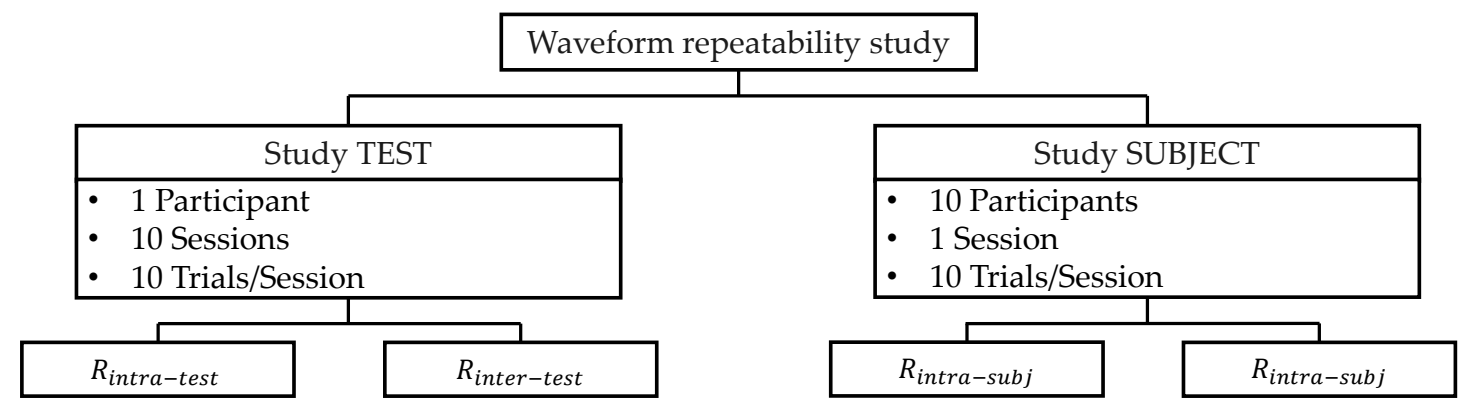

Figure 1. Summary of the waveform repeatability study divided into the study of intra- inter-test and the study of intra- and inter-subject.

\section{Results}

\subsection{Signal Waveforms}

Signal waveforms of the study TEST, for the intra- and inter-test repeatability are presented in Figure 2. For the intra-test study, the participant was able to produce ten similar trials for each session. Moreover, for the inter-test study, high similarities appeared between the sessions, particularly for the longitudinal acceleration and the vertical force. 

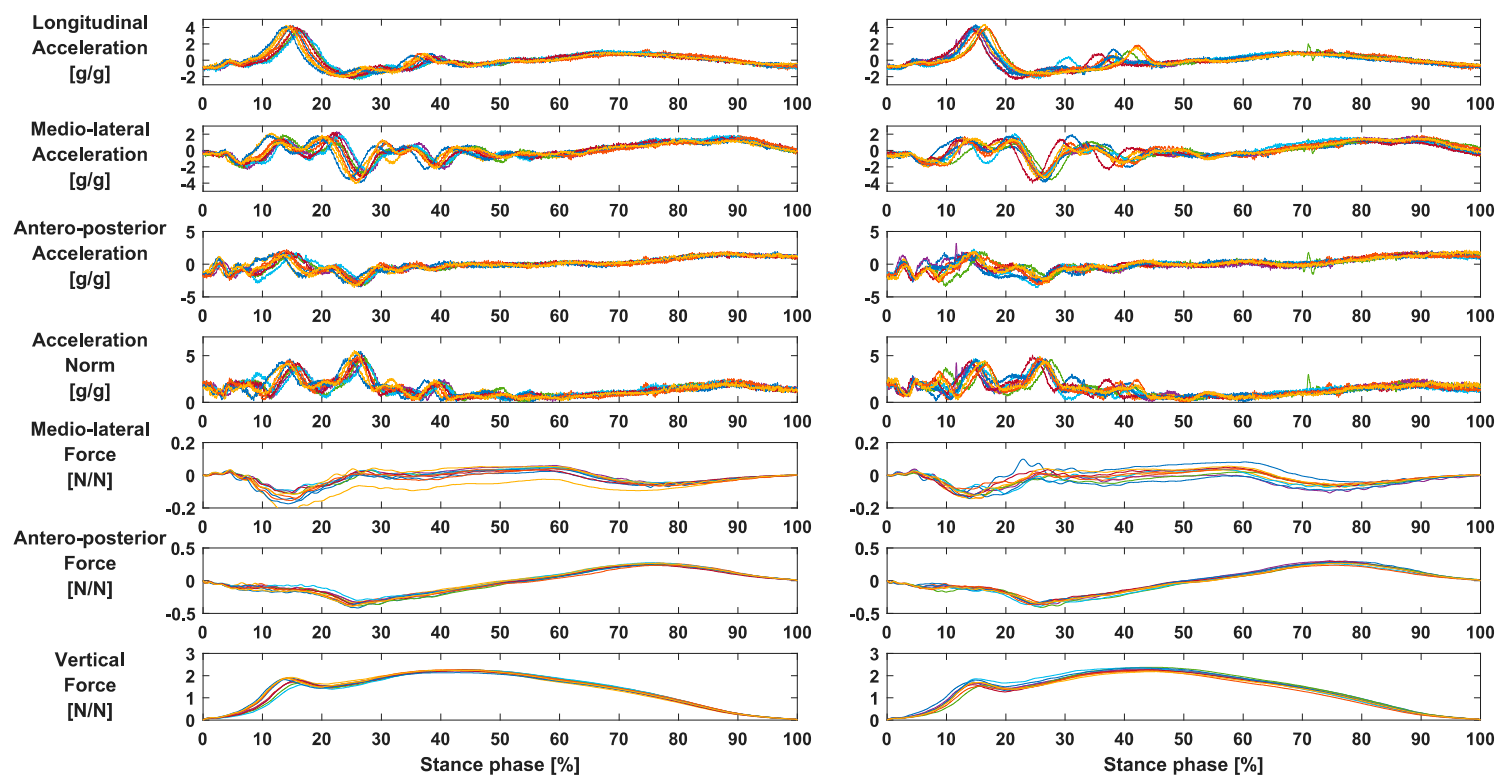

Figure 2. Normalized running signals for acceleration and force during stance for the study TEST. Results for the intra-test repeatability (ten trials of the participant over one session-left) and for the inter-test repeatability (one trial of each session-right).

The signal waveforms of the study SUBJECT, for the intra- and inter-subject repeatability, are presented in Figure 3. For the intra-subject study, all the participants were able to produce ten similar trials. However, waveforms for inter-subject repeatability presented higher variability between participants, especially for medio-lateral accelerations and medio-lateral forces. The most important difference between the participants appeared during the first half of the stance phase. Acceleration waveforms also presented a specific variability between $0 \%$ and $30 \%$ of the stance phase.
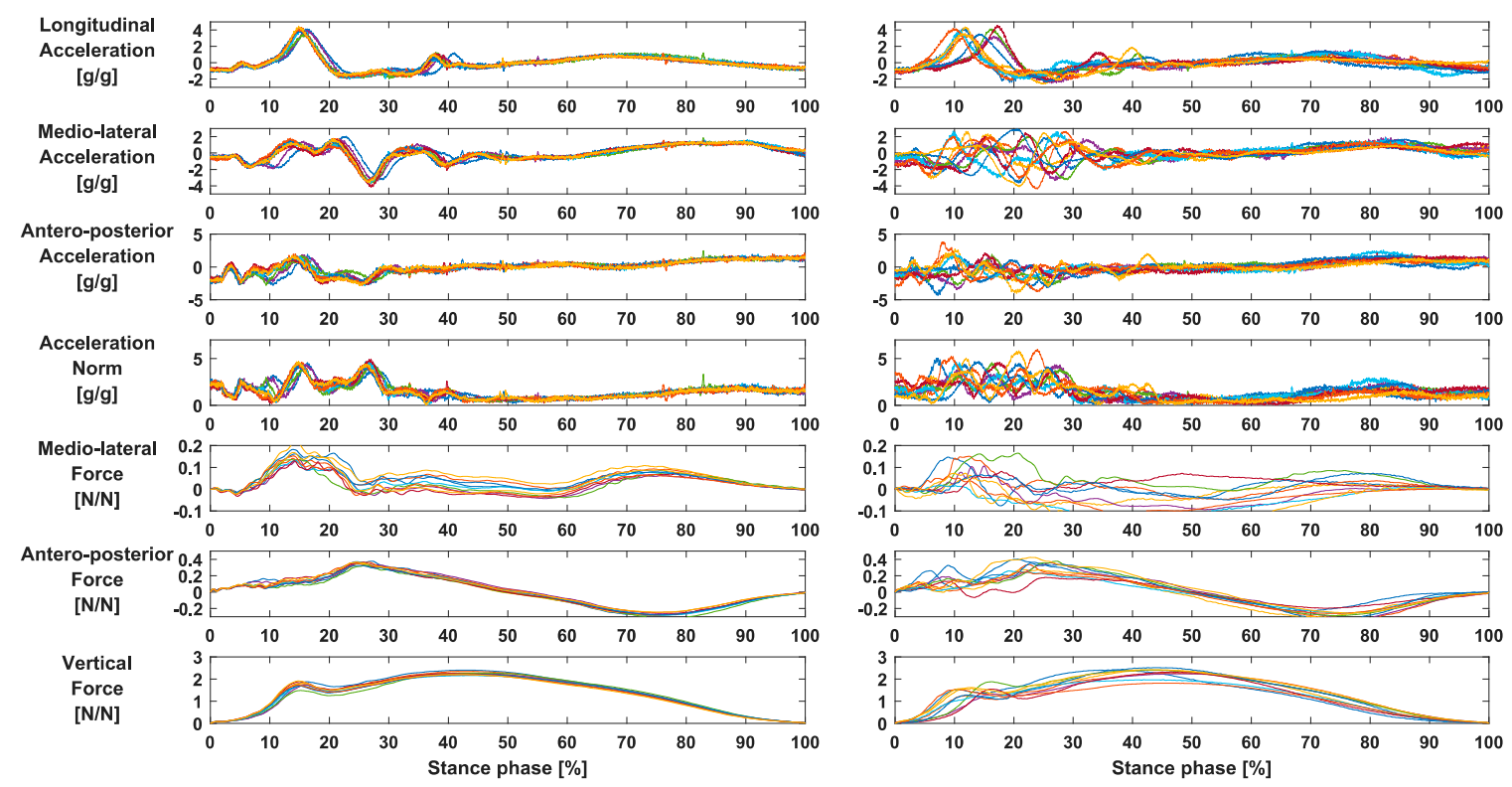

Figure 3. Normalized running signals for acceleration and force during stance for the study SUBJECT. Results for the intra-subject repeatability (ten trials of one participant-left) and for the inter-subject repeatability (one trial of each of the ten participants - right).

\subsection{Signal Repeatability}

Results from the coefficients of multiple correlation for both intra- and inter-test repeatability and intra- and inter-subject repeatability are presented in Table 1. Results highlight the waveform observations. For the study TEST, intra- and inter-test repeatability presented good repeatability for 
each axis of the accelerometer and the force platform. The correlations were better for the longitudinal acceleration and the antero-posterior and vertical forces. For the study SUBJECT, intra-subject repeatability was good for all the signals with a better repeatability observed for the longitudinal acceleration and the vertical and antero-posterior forces. However, inter-subject repeatability was good only for the case of the longitudinal acceleration and the vertical and antero-posterior forces. The poorest repeatability was observed for the medio-lateral axis of both measurement systems.

Table 1. Coefficient of multiple correlation for intra- and inter-subject and test repeatability for longitudinal acceleration (Lon Acc), medio-lateral acceleration (M-L Acc), antero-posterior acceleration (A-P Acc), acceleration norm (Acc Norm), medio-lateral force (M-L Force), antero-posterior force (A-P Force) and vertical force (Vert Force).

\begin{tabular}{lccccccc}
\hline & Lon Acc & M-L Acc & A-P Acc & Acc Norm & M-L Force & A-P Force & Vert Force \\
\hline$R_{\text {intra-test }}$ & 0.973 & 0.952 & 0.946 & 0.942 & 0.913 & 0.995 & 0.997 \\
$R_{\text {inter-test }}$ & 0.937 & 0.905 & 0.918 & 0.898 & 0.854 & 0.991 & 0.994 \\
$R_{\text {intra-subj }}$ & 0.959 & 0.885 & 0.912 & 0.919 & 0.898 & 0.990 & 0.993 \\
$R_{\text {inter-subj }}$ & 0.808 & 0.600 & 0.735 & 0.775 & 0.617 & 0.956 & 0.973 \\
\hline
\end{tabular}

\section{Discussion}

In the case of intra- and inter-test repeatability (study TEST), the proposed study highlights the possibility for a specific participant to produce and reproduce signals for GRF and tibial acceleration. Coefficients of multiple correlation present a good repeatability for each axis of the force and acceleration. This result suggests that typical acceleration and force waveforms can be extracted during running based on a repeatable and reproducible protocol.

For the intra-subject repeatability (study SUBJECT), the results show a similar trend. Each participant generates their own typical acceleration and force waveform according to their preferred speed and equipment. For inter-subject repeatability, coefficients of multiple correlation present a good repeatability for vertical and anteroposterior forces, as well as for longitudinal acceleration. These results validate the second hypothesis: a typical acceleration signal for an amateur runner population can be outlined. However, coefficients of multiple correlation present low repeatability for the mediolateral and antero-posterior accelerations. Consequently, no typical signal can be outlined for these signals. Nonetheless, acceleration signals present a high variability for the first part of the stance phase ( $0 \%-30 \%$ ) caused by the impact of the foot (Figure 2). Indeed, if the signal presents a similar shape, the first part indicates temporal differences. The first acceleration peak reaches its maximum between $10 \%$ and $20 \%$ of the stance phase depending on the participant. Moreover, the width of this peak differs according to the participant. Thus, if the coefficient of multiple correlation presents a low repeatability, this value remains influenced by this main difference. If, however, the signals appear to have a similar shape, the time lag of the first peak is more likely related to the various running speeds adopted by the participants. This relationship between stance time, running speed and time to the first peak has already been observed in the study by Lafortune [14]. Moreover, participants wore different running shoes, with various drops and materials, thus affecting the temporality of the impact through the body. These results could also explain why longitudinal acceleration presents a lower coefficient than vertical or antero-posterior forces.

Considering the inter-subject repeatability, the lowest coefficients of multiple correlation were observed for both accelerometer and force along the medio-lateral axis. Indeed, the variability of the medio-lateral force has already been observed in the literature [5]. This variability is particularly sensitive to the kinematics of the foot: rear-foot strikers show a medio-lateral force more variable than mid-foot strikers. If this study shows that it is possible to extract a typical acceleration signal for the amateur runner, acceleration signals present a sensitivity to the running speed, the shoes and the running pattern. Therefore, acceleration signals remain similar despite this temporal offset, but a future study will have to focus on these parameters, which, apparently, influence the acceleration signals.

More generally, this study quantifies the variability of signal from accelerometer during running and shows that it is possible to extract similar signals from these sensors, even if the use of an accelerometer still presents some limitations. Indeed, the orientation of the sensors reached during 
the mounting add a supplementary bias to the measurement, even if inter-test repeatability was validated. However, the sensors' proposed large band measurement (i.e., full scale, frequency) allows for measuring dynamic information adapted to help one understand the link between the force and acceleration measurement during running. This description could be used in future studies conducted in realistic conditions.

\section{Conclusion}

Amateur runners were able to produce and reproduce similar accelerometric and force waveforms during running following a specific protocol. Each runner holds their own typical waveform during running. Moreover, this study highlights that it was also possible to extract a typical accelerometric and force waveform for a group of amateur runners. Acceleration signals are influenced by the speed, shoes or pattern, specifically the part linked to the impact dampening.

Acknowledgments: The authors wish to thank all the participants in this study.

Conflicts of Interest: The authors declare no conflict of interest.

\section{References}

1. Girard, O.; Millet, G.P.; Slawinski, J.; Racinais, S.; Micallef, J.P. Changes in running mechanics and springmass behaviour during a 5-km time trial. Int. J. Sports Med. 2013, 34, 832-840.

2. Hreljac, A. Impact and Overuse Injuries in Runners. Med. Sci. Sports Exerc. 2004, 36, 845-849.

3. Riley, P.O.; Dicharry, J.; Franz, J.; Croce, U. Della; Wilder, R.P.; Kerrigan, D.C. A kinematics and kinetic comparison of overground and treadmill running. Med. Sci. Sports Exerc. 2008, 40, 1093-1100.

4. Lieberman, D.E.; Venkadesan, M.; Werbel, W.A.; Daoud, A.I.; Dandrea, S.; Davis, I.S.; Mangeni, R.O.; Pitsiladis, Y. Foot strike patterns and collision forces in habitually barefoot versus shod runners. Nature 2010, 463, 531-535.

5. Cavanagh, P.R.; Lafortune, M.A. Ground reaction forces in distance running. J. Biomech. 1980, 13, 397-406.

6. Giandolini, M.; Pavailler, S.; Samozino, P.; Morin, J.B.; Horvais, N. Foot strike pattern and impact continuous measurements during a trail running race: proof of concept in a world-class athlete. Footwear Sci. 2015, 7, 127-137.

7. Camomilla, V.; Bergamini, E.; Fantozzi, S.; Vannozzi, G. Trends supporting the in-field use of wearable inertial sensors for sport performance evaluation: A systematic review. Sensors 2018, 18, 873.

8. Friesenbichler, B.; Stirling, L.M.; Federolf, P.; Nigg, B.M. Tissue vibration in prolonged running. J. Biomech. 2011, 44, 116-120.

9. Giandolini, M.; Poupard, T.; Gimenez, P.; Horvais, N.; Millet, G.Y.; Morin, J.B.; Samozino, P. A simple field method to identify foot strike pattern during running. J. Biomech. 2014, 47, 1588-1593.

10. Thiel, D. V.; Shepherd, J.; Espinosa, H.G.; Kenny, M.; Fischer, K.; Worsey, M.; Matsuo, A.; Wada, T. Predicting Ground Reaction Forces in Sprint Running Using a Shank Mounted Inertial Measurement Unit. Proceedings 2018, 2, 199.

11. Hunter, J.P.; Marshall, R.N.; McNair, P.J. Relationships between ground reaction force impulse and kinematics of sprint-running acceleration. J. Appl. Biomech. 2005, 21, 31-43.

12. Kadaba, M.P.; Ramakrishnan, H.K.; Wootten, M.E.; Gainey, J.; Gorton, G.; Cochran, G.V.B. Repeatability of kinematic, kinetic, and electromyographic data in normal adult gait. J. Orthop. Res. 1989, 7, 849-860.

13. Schache, A.G.; Blanch, P.D.; Rath, D.A.; Wrigley, T. V.; Starr, R.; Bennell, K.L. Intra-subject repeatability of the three dimensional angular kinematics within the lumbo-pelvic-hip complex during running. Gait Posture 2002, 15, 136-145.

14. Lafortune, M.A. Three-dimensional acceleration of the tibia during walking and running. J. Biomech. 1991, $24,877-886$.

(C) 2020 by the authors. Licensee MDPI, Basel, Switzerland. This article is an open access article distributed under the terms and conditions of the Creative Commons Attribution (CC BY) license (http://creativecommons.org/licenses/by/4.0/). 